\title{
The Efficacy of Pharmacotherapy Combined with Short-Term Mindfulness Therapy on the Reduction of Migraine Disability and Improvement of Sleep Quality
}

\author{
Hossein Hosseinpour ${ }^{1}$, Mehdi Akbari ${ }^{*}$, Jafar Hassani ${ }^{1}$, Fatemeh Zargar $^{2}$ \\ ${ }^{1}$ Department of Clinical Psychology, Faculty of Psychology and Education, Kharazmi University, Tehran, Iran \\ ${ }^{2}$ Department of Psychiatry, Faculty of Medicine, Isfahan University of Medical Sciences, Isfahan, Iran
}

\begin{tabular}{|ccc} 
& Article Info: & \\
Received: 17 Sep 2019 & Revised: 28 Oct 2019 & Accepted: 2 Dec 2019
\end{tabular}

\section{ABSTRACT}

Introduction: Migraine headaches are one of the most common types of headaches that can cause significant dysfunction. Sleep disturbances are common symptoms and one of the stimulating factors of migraine. The goal of this study was to the comparison of the efficacy of pharmacotherapy combined with short-term mindfulness-based therapy and pharmacotherapy on the reduction of migraine disability and improvement of sleep quality in women with migraines. Materials and Methods: The research was a quasi-experimental pretest-posttest design with the control group. The statistical population of this study included all women with migraine attending to a psychosomatic clinic in Isfahan city from August to November of 2018. Twenty-eight patients who were diagnosed with migraines were randomly assigned to the experimental group (pharmacotherapy combined with short-term mindfulnessbased therapy) and control group (pharmacotherapy). Migraine Disability Assessment (MIDAS) and Pittsburgh Sleep Quality Index (PSQI) were administered to collect data. Results: Pharmacotherapy combined with short-term mindfulness-based therapy led to a significant reduction of migraine disability and intensity, improvement of sleep efficiency and quality, reduction of sleep latency, and daytime dysfunction compared to the control group. However, migraine frequency and other domains of sleep quality did not differ between the groups. Conclusion: These data suggest that short-term mindfulness-based therapy besides pharmacotherapy could be effective for improving migraine disorder and sleep quality in patients with migraines.

\section{Key words:}

1. Headache

2. Migraine Disorders

3. Sleep

4. Mindfulnes

5. Drug Therapy

*Corresponding Author: Mehdi Akbari

E-mail:akbari.psy@gmail.com 


\title{
اثربخشـى دارودرمانى تر كيبشده با درمان كوتاهمدت ذهن آَّاهى در كاهش ناتوانى ناشى از ميگرن و بهبود كيفيت خواب
}

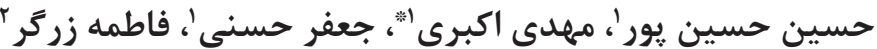 \\ آرووه روانشناسى بالينى، دانشكده روانشناسى و علوم تربيتى، دانشًاه خوارزمى، تهران، ايران \\ rَرَوه روانيزشكى، دانشكده يزشكى، دانشَاه علوم يزشكى اصفهان، اصفهان، ايران
}

اطلاعات مقاله:

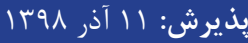

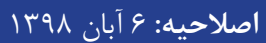

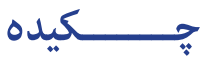

مقدمص: سردردهاى ميكرنى يكى از شايعترين انواع سردرد مىباشد كه باعث افت عملكرد قابل توجهى

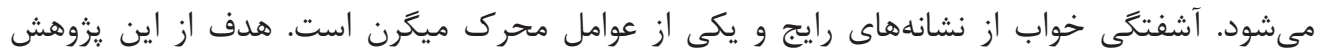

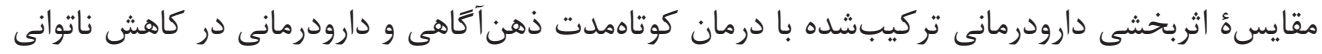

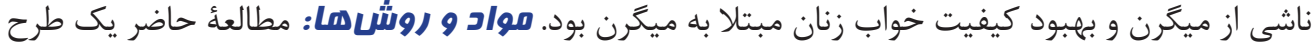

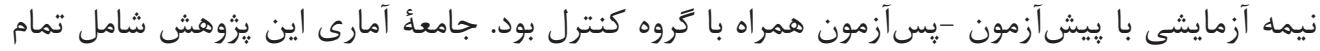

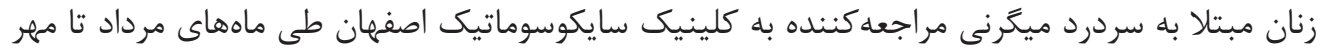

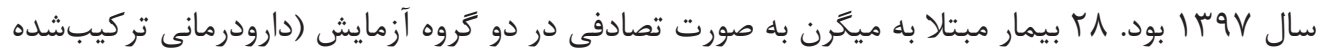

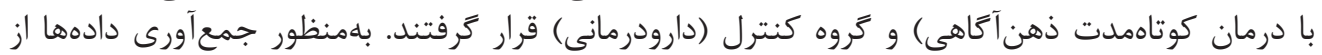

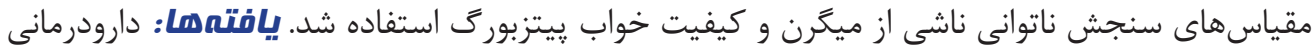

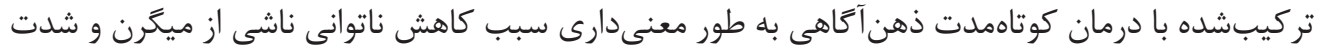

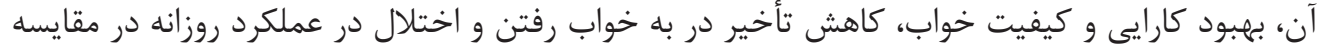

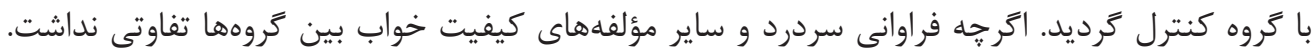

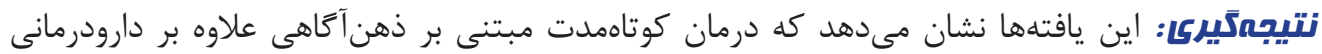

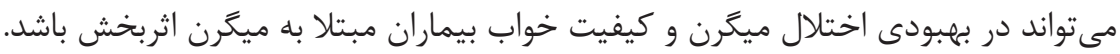

كليد وازهها:

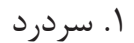

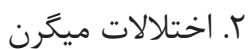

r.

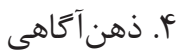

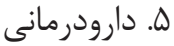

" نويسنده مسئول: مهدى اكبرى :

آدرس الكترونيكى: akbari.psy@gmail.com 


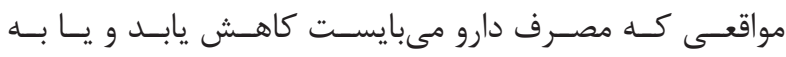

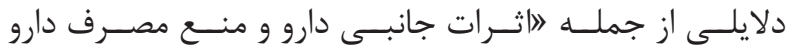

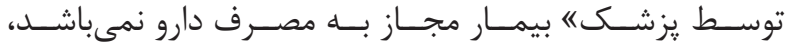

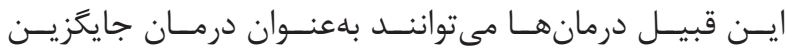

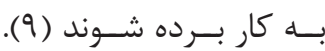

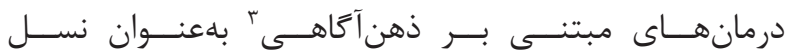

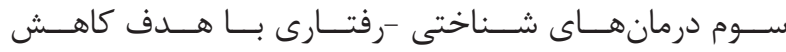

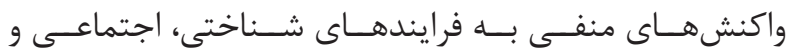

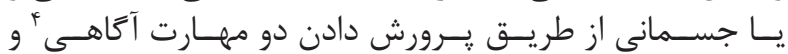

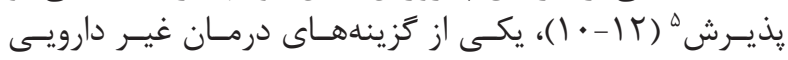

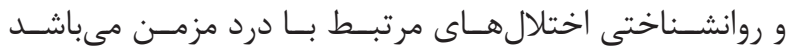

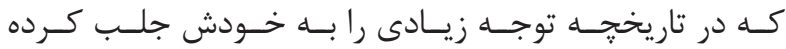

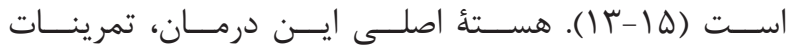

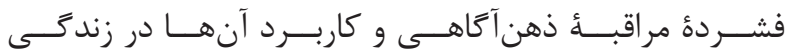

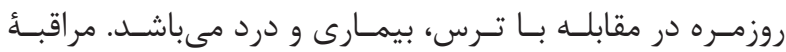

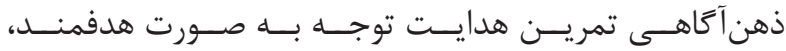

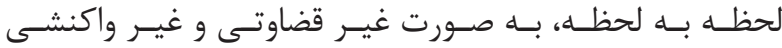

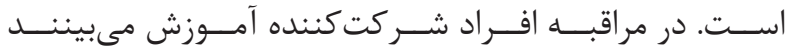

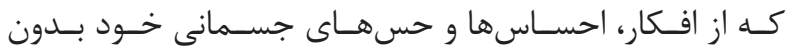

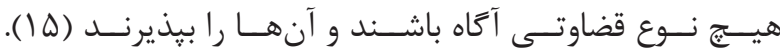

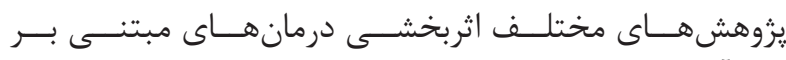

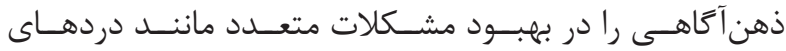

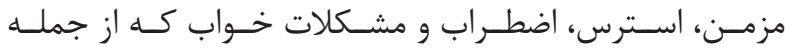

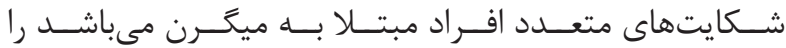
تأييـــد كردهانـــ (19، IV)

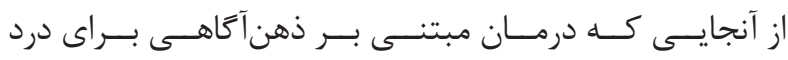

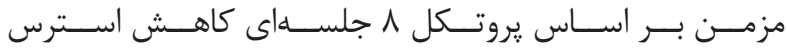

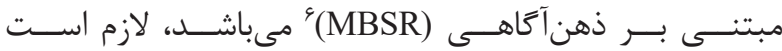

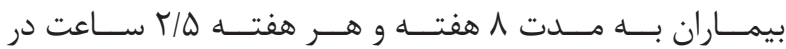

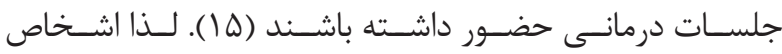

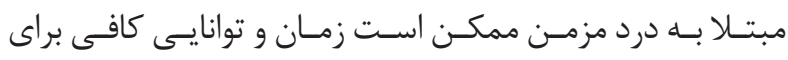

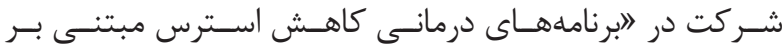

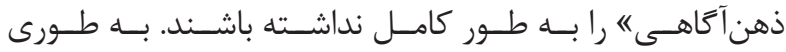

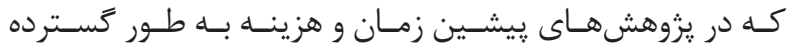

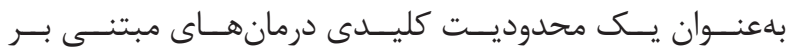

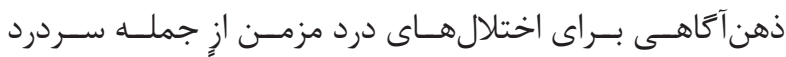

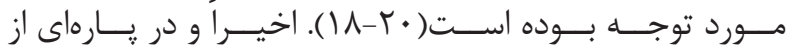

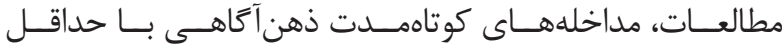

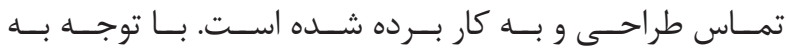

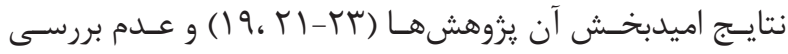

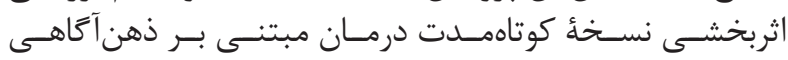

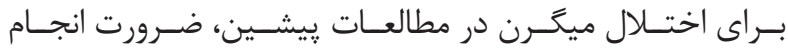

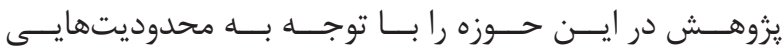

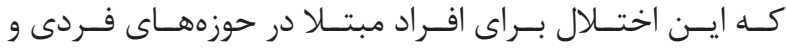

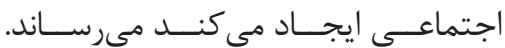

${ }^{1}$ Headache and migraine

${ }^{2}$ Biofeedback

${ }^{3}$ Mindfulness

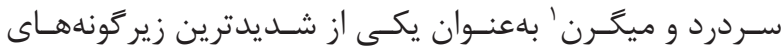

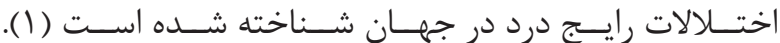

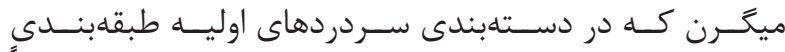

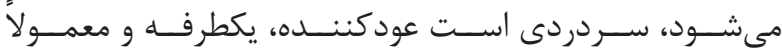

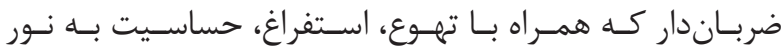

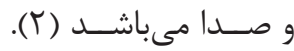

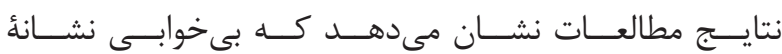

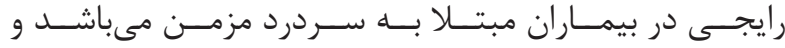

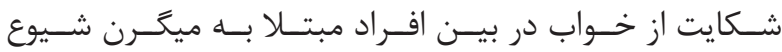

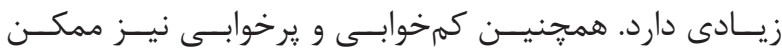

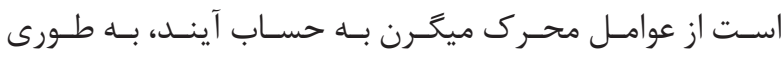

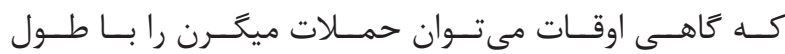

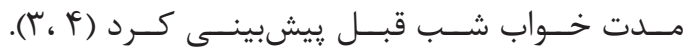

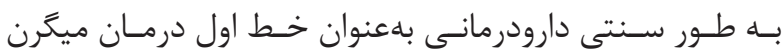

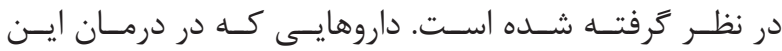

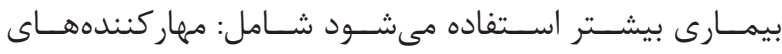

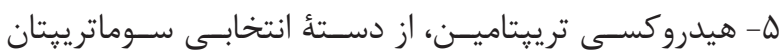

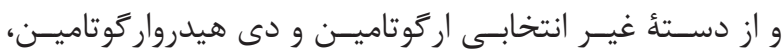

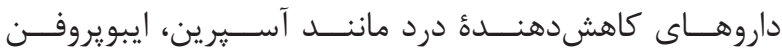

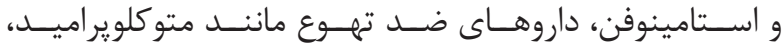

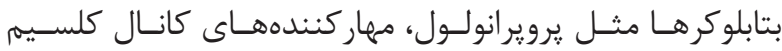

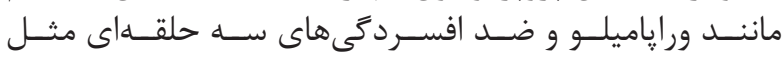

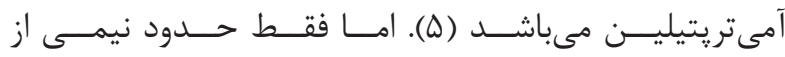

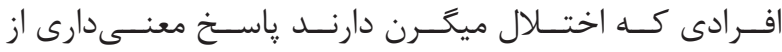

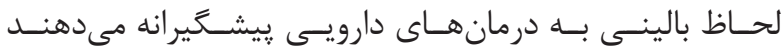

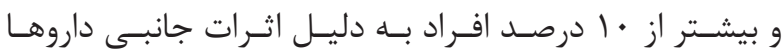

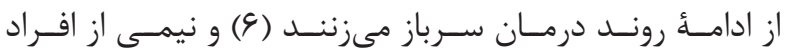

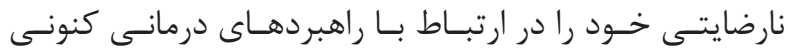

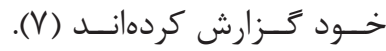

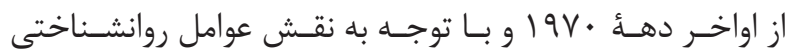

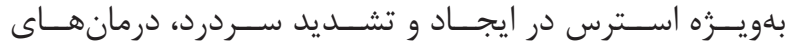

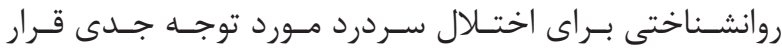

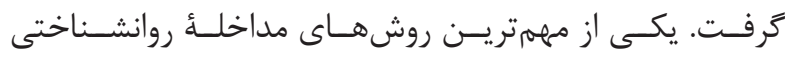

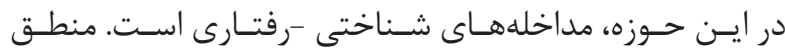

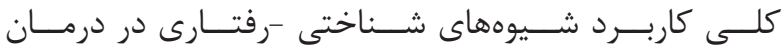

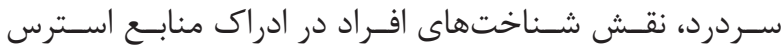

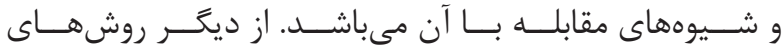

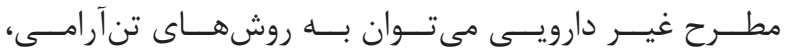

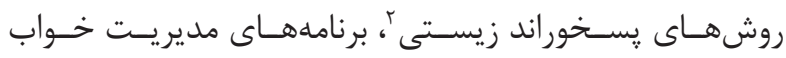

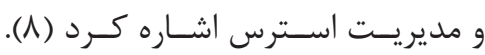

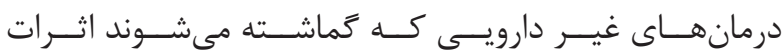

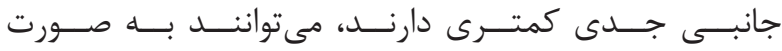

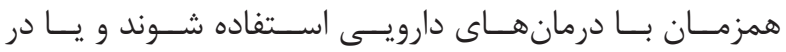

${ }^{4}$ Awareness

${ }^{5}$ Acceptance

${ }^{6}$ Mindfulness based stress reduction 


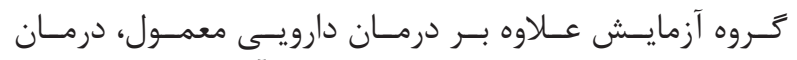

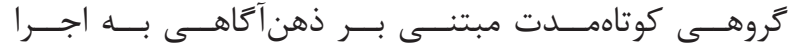

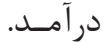

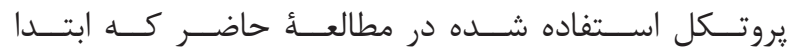

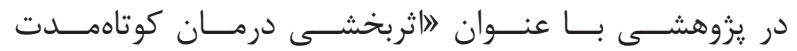

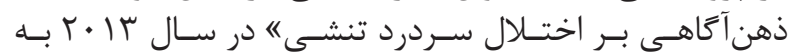

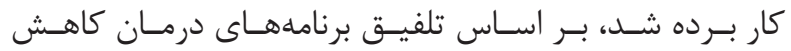

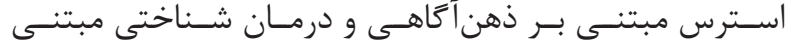

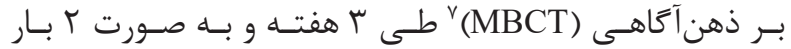

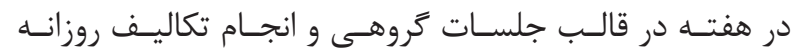

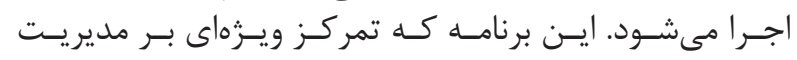

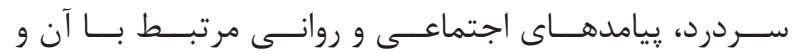

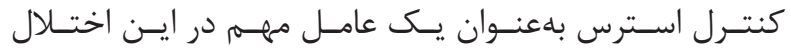

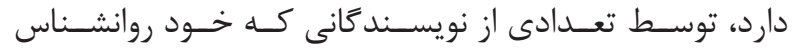

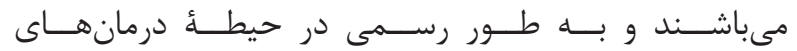

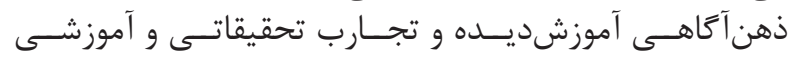

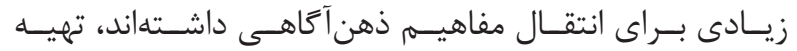

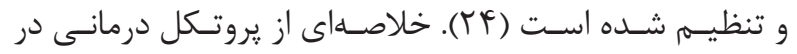

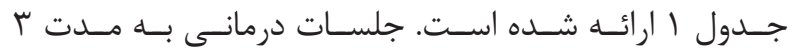

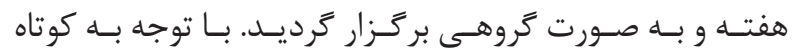

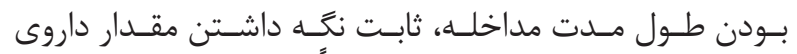

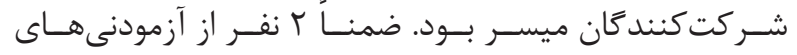

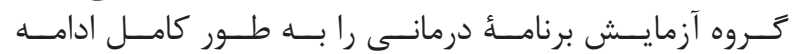

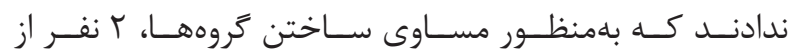

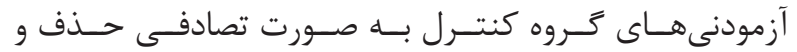

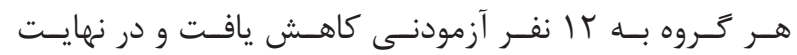

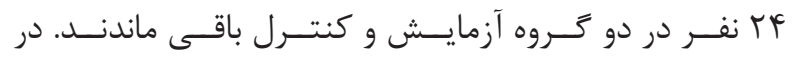

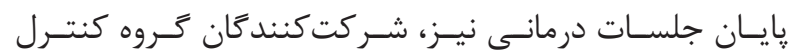

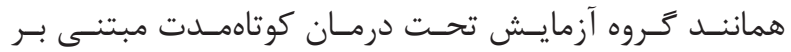

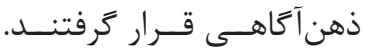

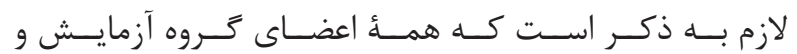

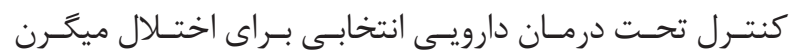

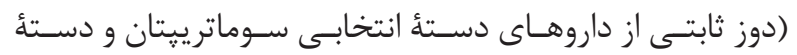

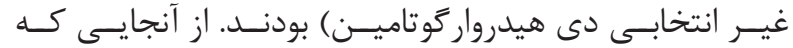

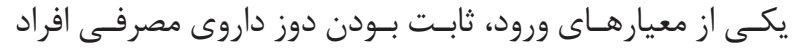

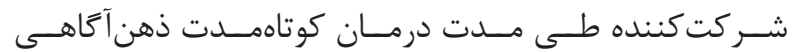

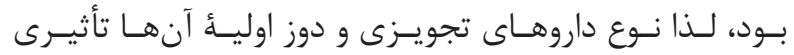

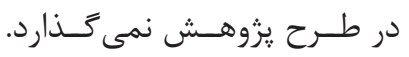

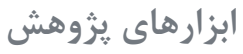

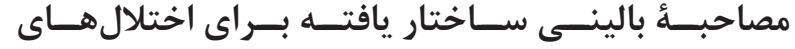

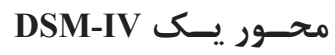

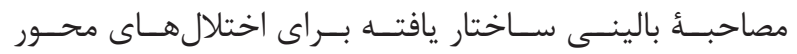

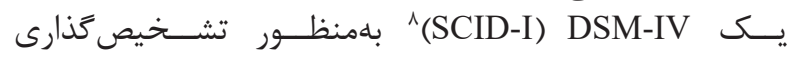

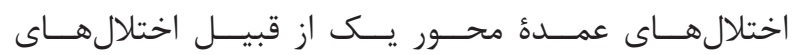

بـا توجـهـ بــه شـيوع بيشـتر اختـلال ميخــرن در جمعيـت زنان

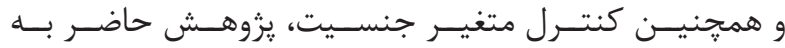

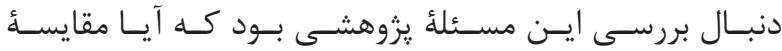

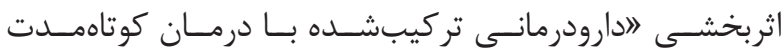

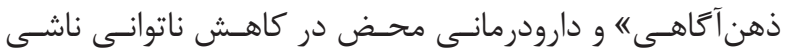

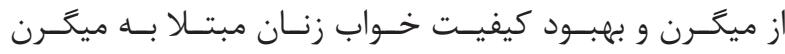

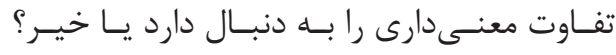

\section{مواد و روشها}

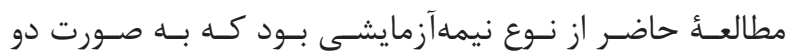

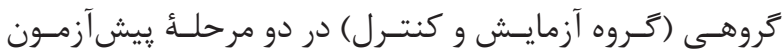

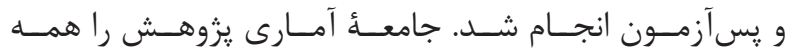

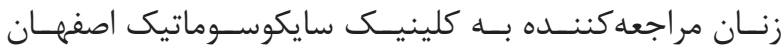

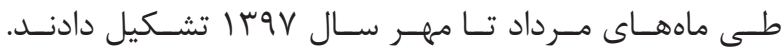

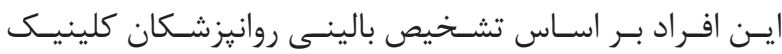

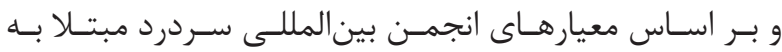

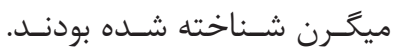

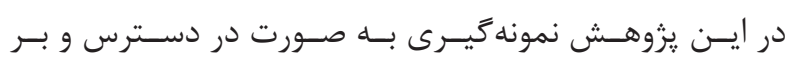

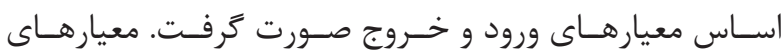

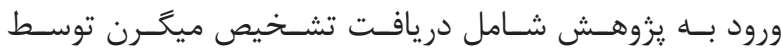

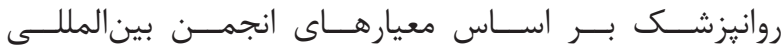

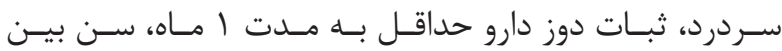

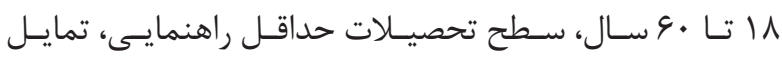

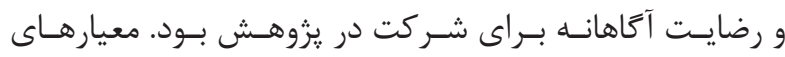

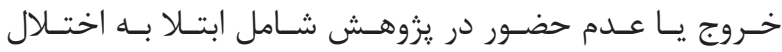

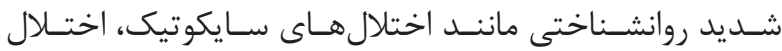

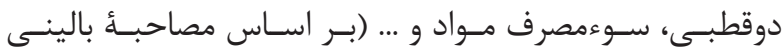

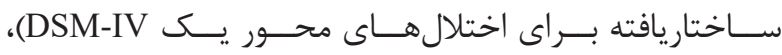

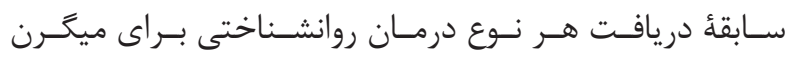

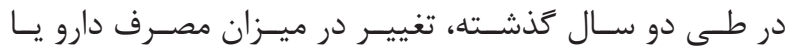

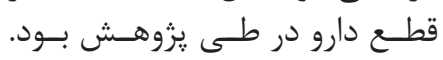

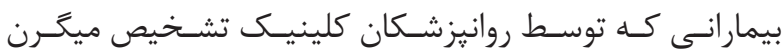

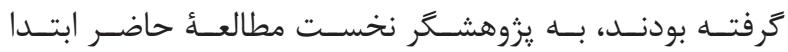

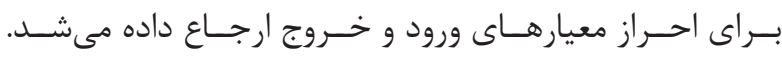

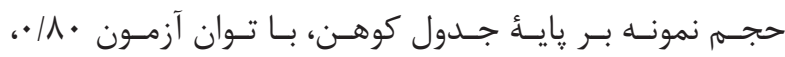

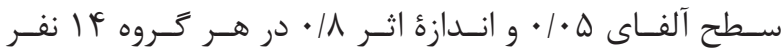

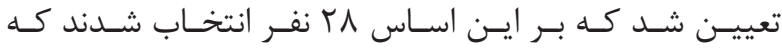

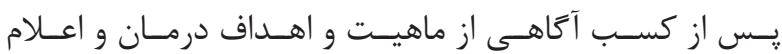

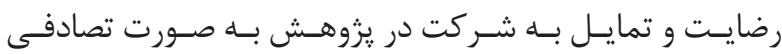

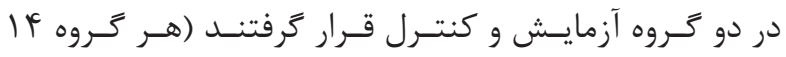

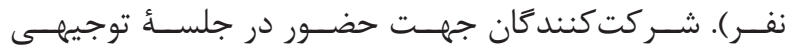

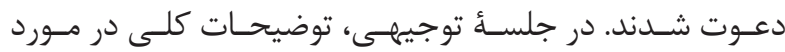

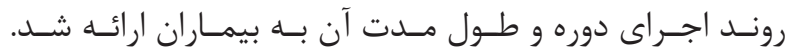

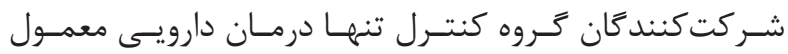

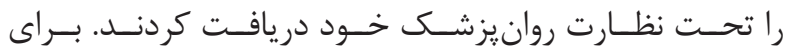

${ }^{7}$ Mindfulness based cognitive therapy

${ }^{8}$ Structural clinical interview for DSM-IV-axis I 
جدول ا- جهار خوب كلى جلسات درمان كوتامدت مبتنى بر ذهن آكاهى.

\begin{tabular}{|c|c|}
\hline محتواى جلسات & جلسات \\
\hline روند بركزارى دوره و طول مدت آن. توضيح كلى در مورد ذهن آكاهى به زبان ساده. فرم & مقدمه \\
\hline 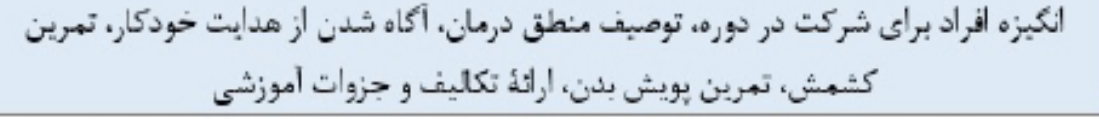 & جلسة | \\
\hline 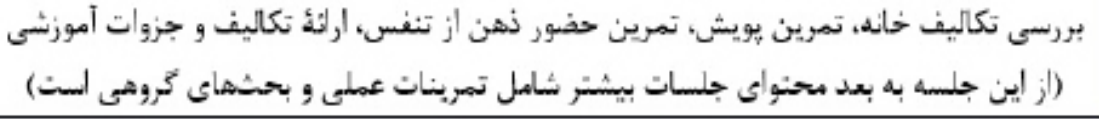 & جلسة r \\
\hline 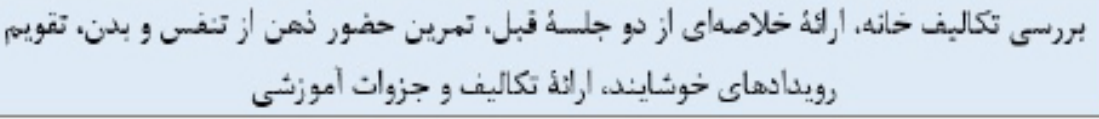 & جلسة ب \\
\hline 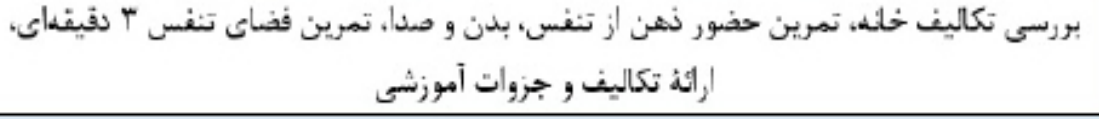 & جلسة F F \\
\hline 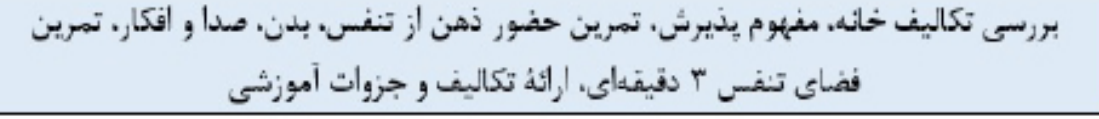 & جلسة هـ \\
\hline 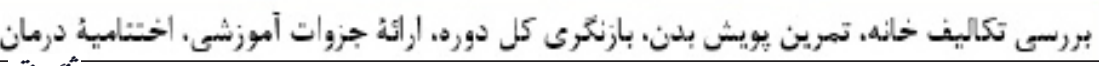 & جلسة 9 - و \\
\hline
\end{tabular}

را در يـك طيـف • ا درجـهـاى مشـخص مى كنــــد كـه عـدد

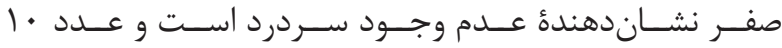

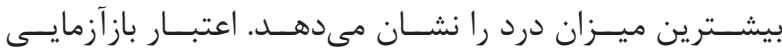

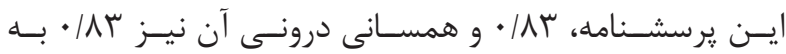

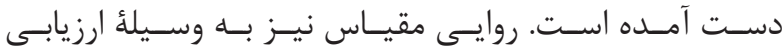

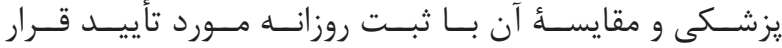

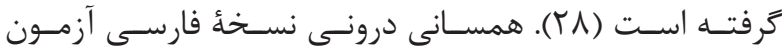

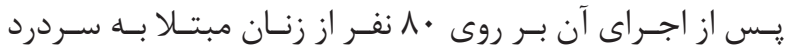

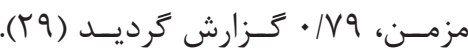

\section{يرسشنامة كيفيت خواب بيتزبورى}

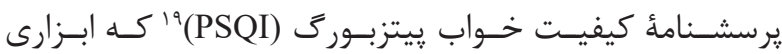

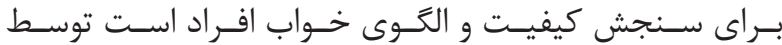

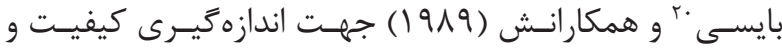

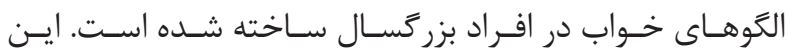

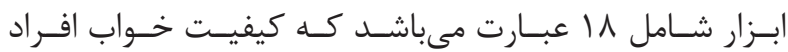

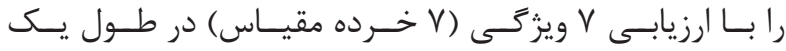

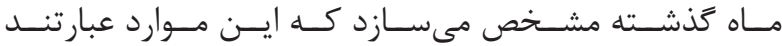

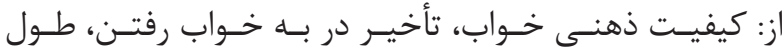

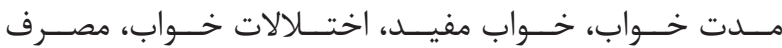

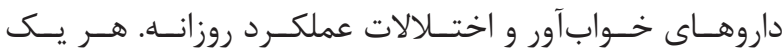

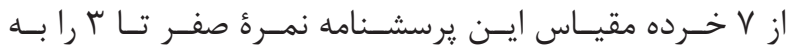

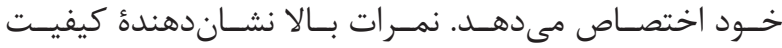

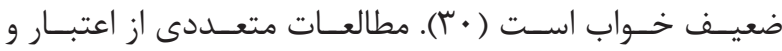

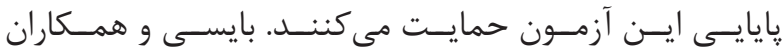

${ }^{9}$ Diagnostic and statistical manual of mental disorders-IV

${ }^{10}$ First

${ }^{11}$ Spitzer

${ }^{12}$ Gibbon

${ }^{13}$ Williams

${ }^{14}$ Tran

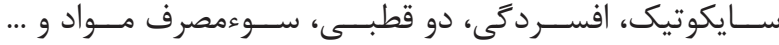

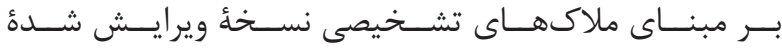

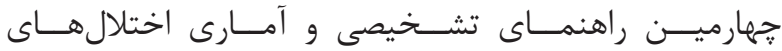

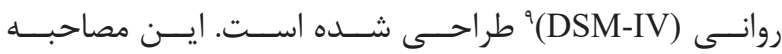

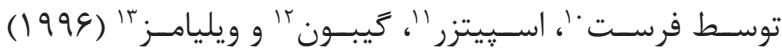

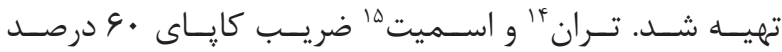

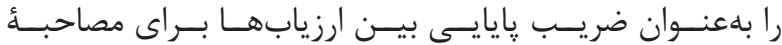

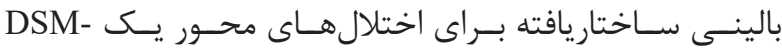
IV

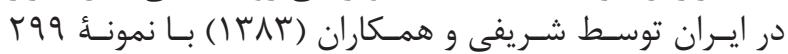

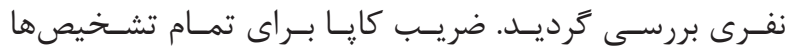

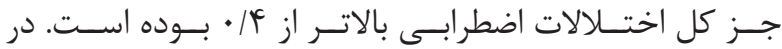

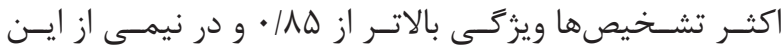

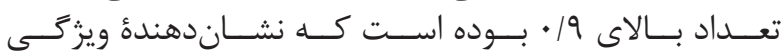

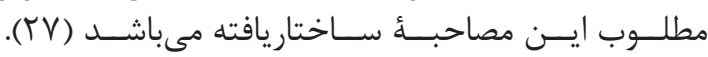

\section{مقياس سنجش ناتوانى ناشى از ميگرن}

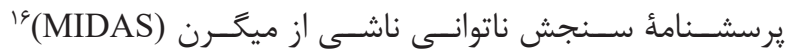

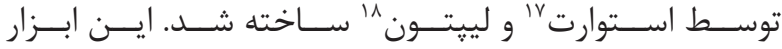

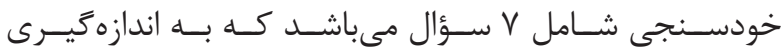

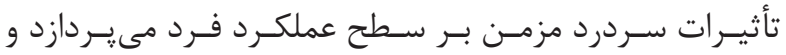

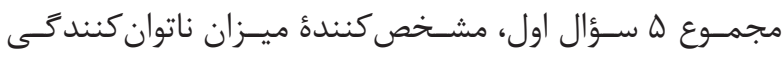

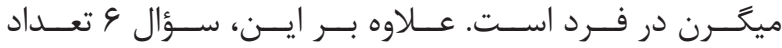

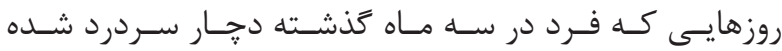

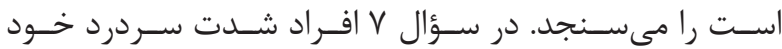

${ }^{15}$ Smith

${ }^{16}$ Migraine disability assessment

${ }^{17}$ Stewart

${ }^{18}$ Lipton

${ }^{19}$ Pittsburgh sleep quality index

${ }^{20}$ Buysse 


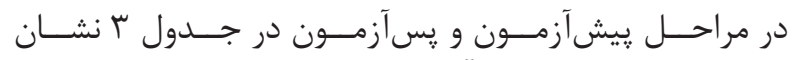

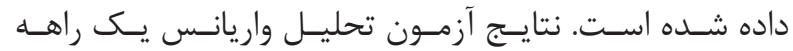

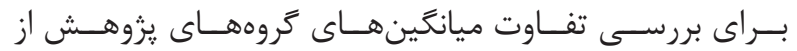

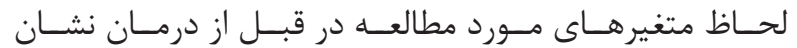

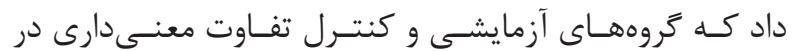

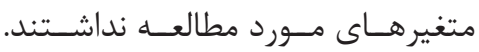

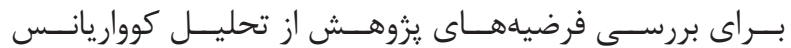

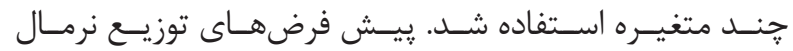

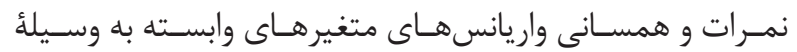

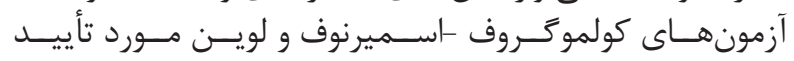

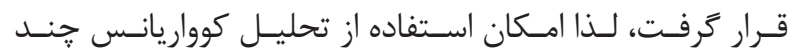

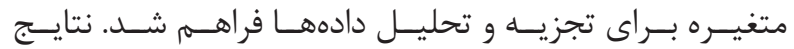

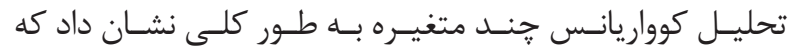

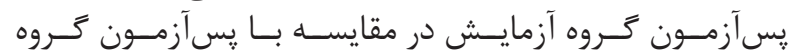

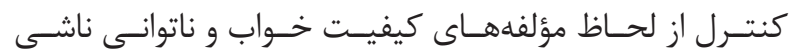

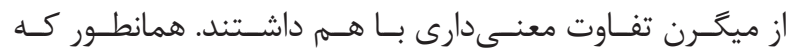

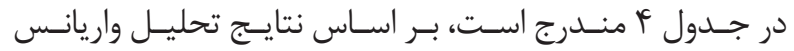

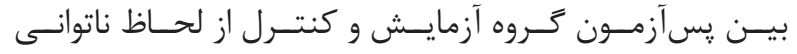

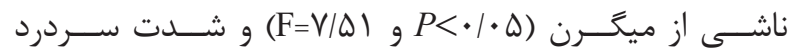

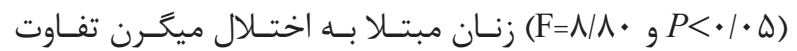

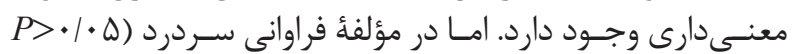

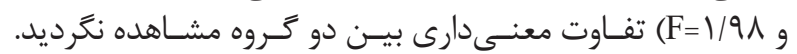

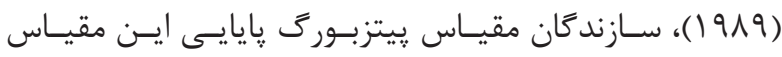

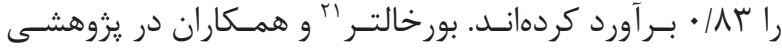

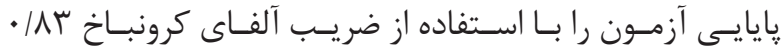

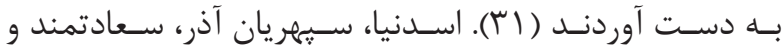

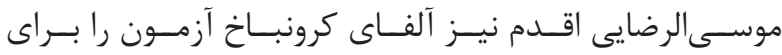

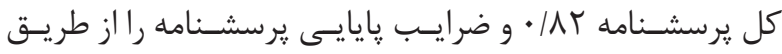

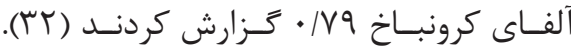
تجزيه و تحليل دادهها

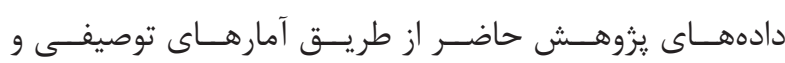

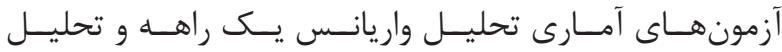

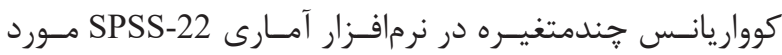

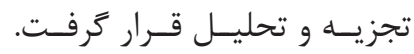
بافتهها

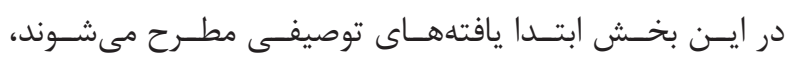

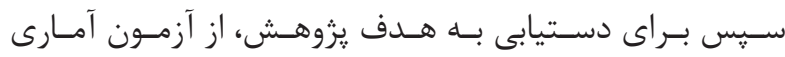

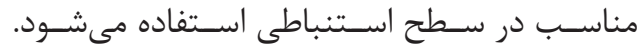
اطلاعات جمعيتشناختى شركت كنندكان شامل سن، ميزان

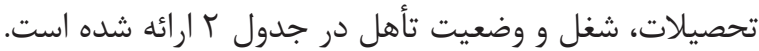

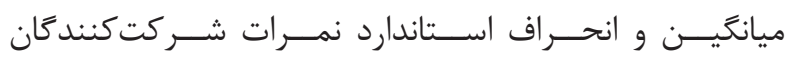

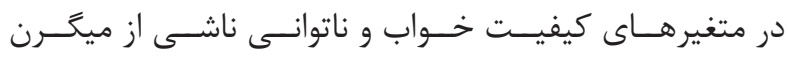
جدول r- اطلاعات جمعيتشناختى شركت كنندًان.

\begin{tabular}{|c|c|c|c|}
\hline "ثروه كثترل & "حروه آزمايش & \multicolumn{2}{|c|}{ وفرثن } \\
\hline F (Fr/Yr) & $Y(\mid 9 / 99)$ & M-YA & \\
\hline$\Gamma(\mid \varphi / F \varphi)$ & $P(T T / T)$ & rq-rq & سين (سال) \\
\hline$r(r \Delta)$ & $\varphi(D \cdot)$ & $F=-\theta$. & تعداه (مرهد) \\
\hline$T(\backslash, / F F)$ & 1 (N/T) & $\Delta 1-\bar{\varphi}$. & \\
\hline & & زير ديبلم & \\
\hline $\begin{array}{r}1(N T) \\
r \quad 1 \in 00\end{array}$ & $+(+)$ & ديلم: & \\
\hline (I)/V) & $T(T Q)$ & نوفى ديبلم & تحصيلات \\
\hline $1(N T)$ & $r(1 / 9 / Y)$ & كارثشنائسي & تعداد (مرحد) \\
\hline$Y(\Delta N Y)$ & $\varphi(\Delta+)$ & 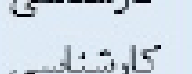 & \\
\hline $1(N T)$ & $1(N T)$ & ارثشد & \\
\hline$Y(Q N Y)$ & $\Psi(Y \Delta)$ & مجترد & وفقعيت ثأهل \\
\hline$\Delta(F Y / V)$ & $9(V \Delta)$ & م-أهل & تعداد (درصد) \\
\hline$r(I F / V)$ & $V(\Delta N T)$ & حانهار & \\
\hline$\Delta(F) / V)$ & roin & شاغل & وضسيت اشتثال \\
\hline$+(+)$ & $1(N)$ & يازتثــي & تعداد (روصد) \\
\hline$\Delta(F) / V)$ & $r(19 / 7)$ & ييكار & \\
\hline
\end{tabular}

${ }^{21}$ Burkhulter 
جدول بـ- ميانكَين و انحراف استاندارد براى نمرات متغيرهاى يُوهش در ييشآزمون و يِ آزمون.

\begin{tabular}{|c|c|c|c|c|c|c|}
\hline \multicolumn{2}{|c|}{ بس أزيوئ } & \multicolumn{2}{|c|}{ 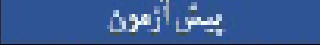 } & \multirow{2}{*}{ "كرو' } & \multirow{2}{*}{ قتخير } & \\
\hline 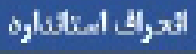 & 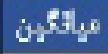 & oflatinat & a & & & \\
\hline d $10 \gamma$ & $111 \%$ & frva & vas & آزيايش & \multirow{2}{*}{ كيليت لمنى كلواب } & \\
\hline$\cdot 159$ & $1, \pi 5$ & .84 & $V / \uparrow)$ & كثترل & & \\
\hline . $h$ & 1 & $2 / 91$ & $\mathrm{~V} \Delta \mathrm{A}$ & آزهايش & \multirow{2}{*}{ تأمثر م, } & \\
\hline$\cdot 199$ & $1,1, \mathrm{Fi}$ & vis & int & كتثرل & & \\
\hline $4 \pi$ & $\cdot / \lambda^{2}$ & $V \cdot \lambda$ &.$/ 91$ & آزعابش & \multirow{2}{*}{ طول فه تخواتي } & \\
\hline$\cdot|V|$ & 1116 & $\mathrm{~V} \cdot \mathrm{S}$ & V/TO & كتثرل & & \\
\hline 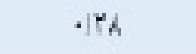 & $.1 \cdot 1$ & +19. & +10. & آزمايثر & \multirow{2}{*}{ توابي عفيك } & \\
\hline 1$] \cdot \gamma$ & .97 & vis & :AT & كتثرل & & \\
\hline . $9 \%$ & Iffi & $.1 \% 4$ & VQA & آزمايثر & \multirow{2}{*}{ الخعالالات خزواب } & \\
\hline , $6 *$ & $1 \pi \pi$ & $\infty 9$ & $v(f)$ & كتثرل & & \\
\hline $11 \% 0$ & . 190 & vir &.$/ 91$ & آزمايثر & \multirow{2}{*}{ 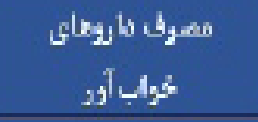 } & \\
\hline $1 / 18$ & - $J \Delta A$ &.$/ 9$. & $\cdot / \Delta$. & كت: & & \\
\hline$\cdot w$ & .46 & .194 &.$(9)$ & آزمايش & \multirow{2}{*}{ إكتالاكت مصاكرى } & \\
\hline $4 \mathrm{Mk}$ & INT & via & 1,95 & كت:ترd & & \\
\hline$T-\alpha$ & 841 & $4 / .3$ & $n+1$ & آزمايش & \multirow{2}{*}{ نعرة كالى 'كيليت يخواب } & \\
\hline Y $|\Delta|$ & Y) AT & Y/A1 & Nfi & كت:رل & & \\
\hline $\mathrm{Terv}$ & TVAT & 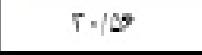 & T./AT & أزعابش & \multirow{2}{*}{ 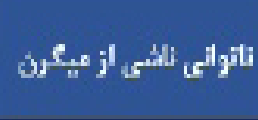 } & \\
\hline $19 \% 9$ & THIAT & IOSY & $r \cdot 1 \cdot h$ & كنئ & & \\
\hline $19 / 04$ & $10 / T 2$ & TQFT & Thes & آزعابش & \multirow{2}{*}{ ق } & \\
\hline IF/AV & $18 \pi \mathrm{T}$ & TYF. & $12 / 41$ & ك5 & & \\
\hline$T+*$ & $\Delta$ & $T / 94$ & 909 & آزعايش & \multirow{2}{*}{ لهدت } & \\
\hline TIT. & $6 / 40$ & 1/Fo & $\Delta y$ & كثترل & & \\
\hline
\end{tabular}

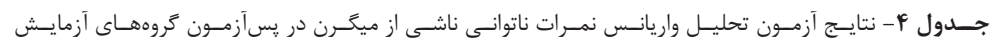

\begin{tabular}{|c|c|c|c|c|c|}
\hline معنى دارى & $\mathbf{F}$ & MS & Df & SS & متغير \\
\hline$\cdot 1 \cdot 1$ & $V / D \mid$ & $1 T \Delta \cdot / 94$ & 1 & $1 T \omega \cdot 194$ & ناتوانى ناشى از ميكّرن \\
\hline.$/ 1 \mathrm{~V}$ & $1 / 91$ & $1 \% q / q \varphi$ & 1 & $\mid r q / q \varphi$ & فراوانى \\
\hline$\cdot / \cdot \Lambda$ & $N / \Lambda$ & $r \cdot 19 r$ & 1 & $r \cdot \mid 9 r$ & 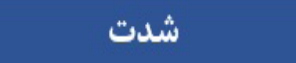 \\
\hline
\end{tabular}

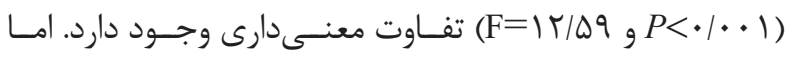

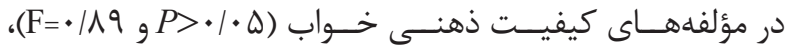

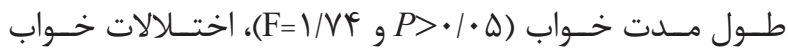

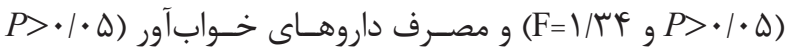

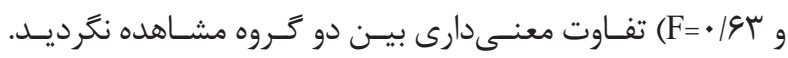

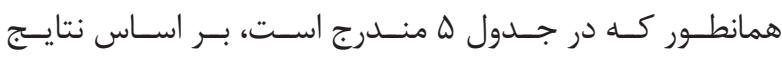

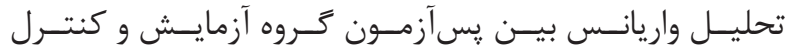

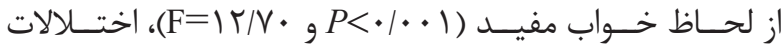

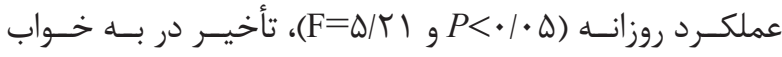

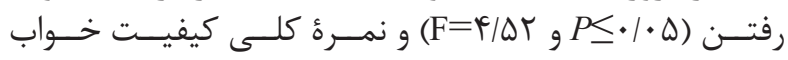


جدول ه- نتايج آزمون تحليل واريانس نمرات كيفيت خواب در يسآزمون كروههاى آزمايش و كنترل.

\begin{tabular}{|c|c|c|c|c|c|}
\hline معثى فارى & $\mathbf{F}$ & MS & df & SS & متغير \\
\hline$\cdot /$ He & $\cdot / 199$ &.$/ 4 r$ & 1 &.$/ 4 r$ & كيفيت ذهنى خواب \\
\hline$+1+\Delta$ & $f / \Delta r$ & $1 / A F$ & 1 & l/NF & تأخير در به خواب رفتن \\
\hline$\cdot / T \cdot$ & $1 / V 4$ & $\cdot / f \cdot$ & 1 & $\cdot / f \cdot$ & طول مدت خواب \\
\hline$* r$ & $I T / V$. & $f / f \wedge$ & 1 & $f / f \wedge$ & خواب مفيد \\
\hline$\cdot / T E$ & $1 / T F$ & $\cdot / 4 r$ & 1 & . / rt & اختنالات خواب \\
\hline 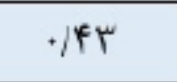 & $+/ 9 \pi$ & $\cdot / \Delta f$ & 1 & $+/ \Delta f$ & مصرف داروهاى خوابآرو \\
\hline$\cdot / \cdot r$ & $\Delta / r)$ & I/rT & 1 & $1 / r r$ & اختلالات عملكرد روزانه \\
\hline$\because \cdots r$ & $1 \pi / 09$ & $f q / 1 f$ & 1 & fq/1f & نمرة كلى كيفيت خواب \\
\hline
\end{tabular}

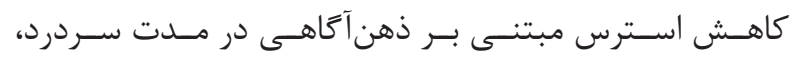

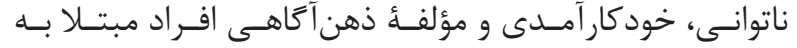

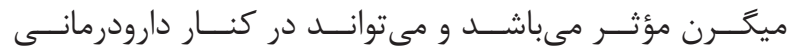

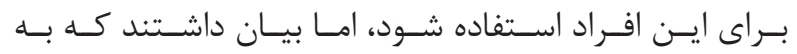

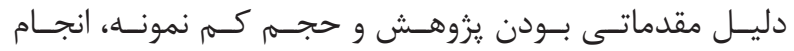

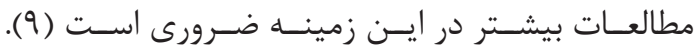

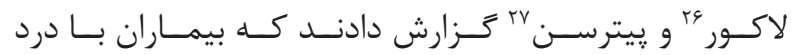

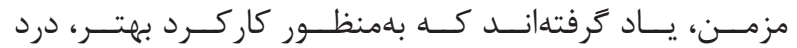

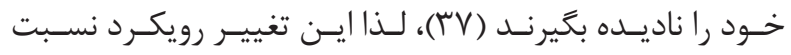

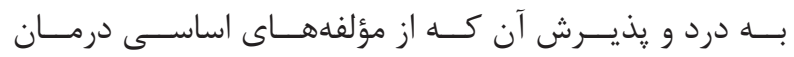

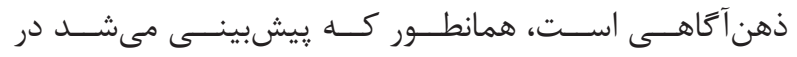

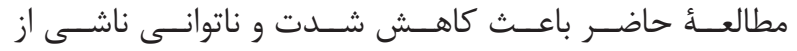

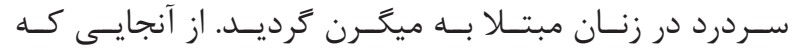

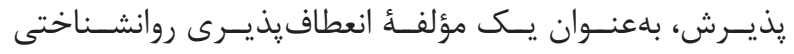

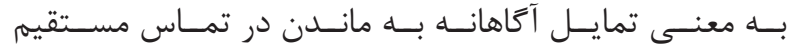

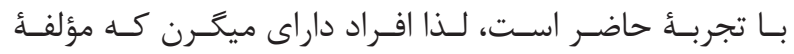

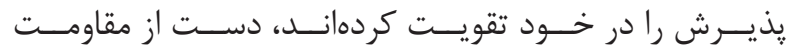

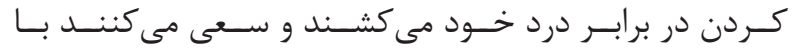

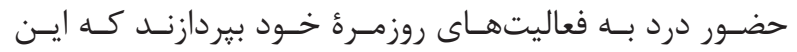

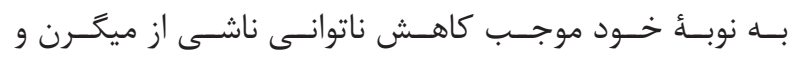

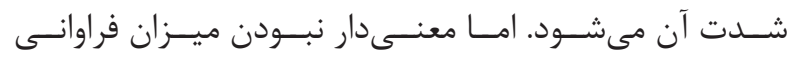

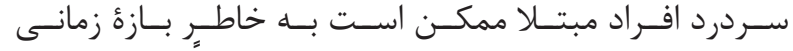

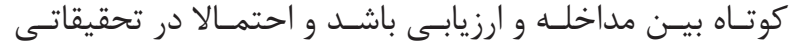

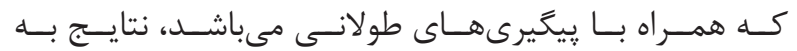

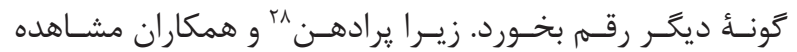

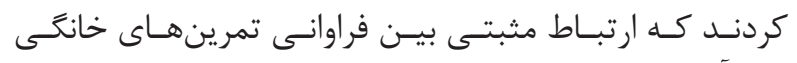

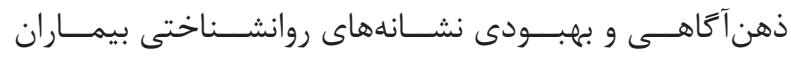

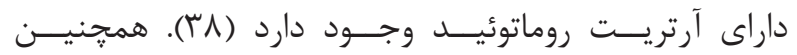

${ }^{22}$ Rosdahl

${ }^{23}$ Cathcart

${ }^{24}$ Schmidt

${ }^{25}$ Wells
بحث و نتيجهَ'

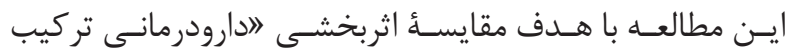

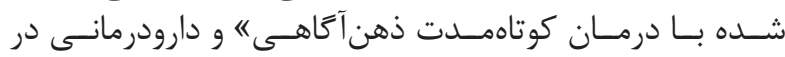

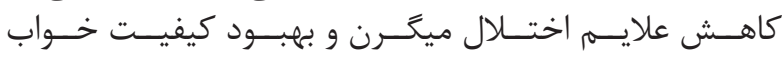

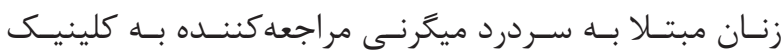

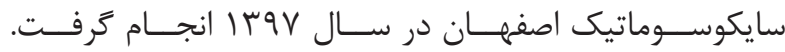

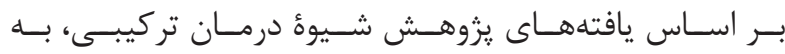

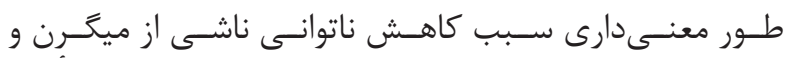

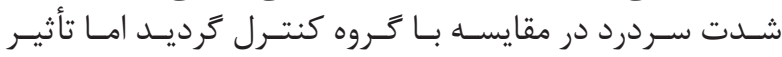

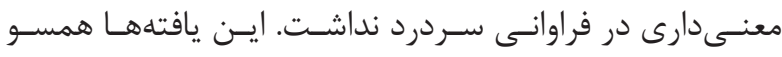

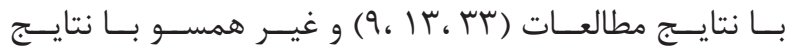

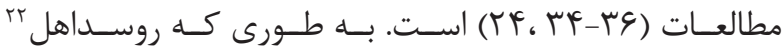

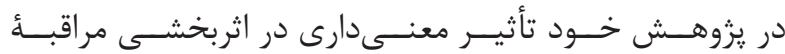

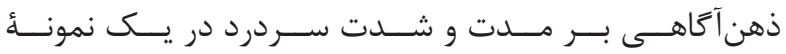

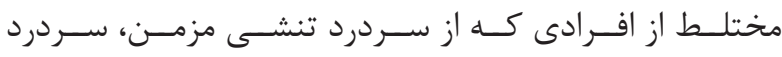

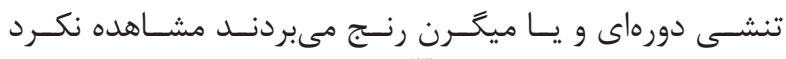

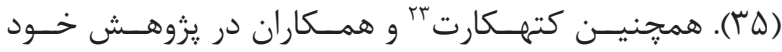

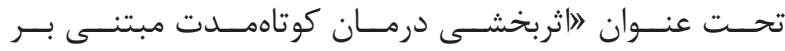

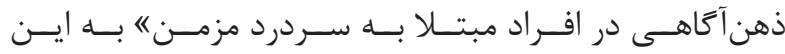

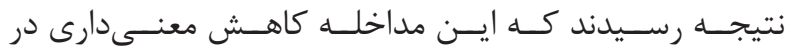

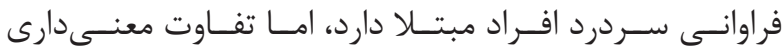

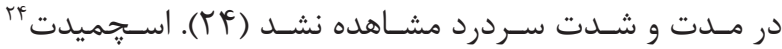

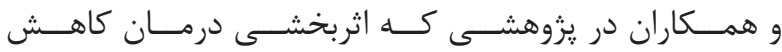

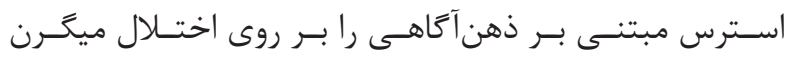

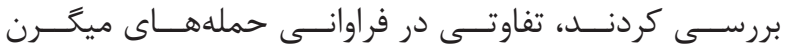

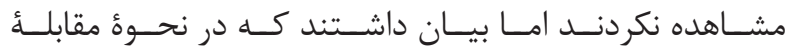

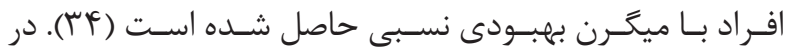

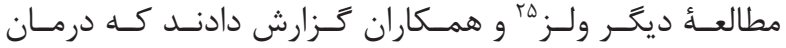

\footnotetext{
${ }^{26}$ La Cour

${ }^{27}$ Petersen

${ }^{28}$ Pradhan
} 


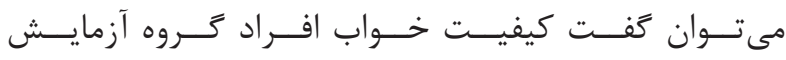

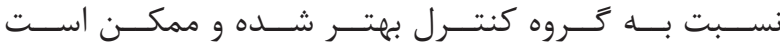

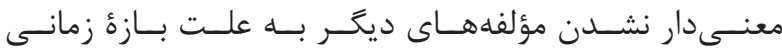

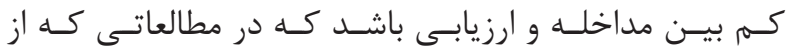

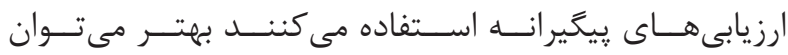

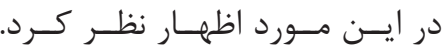

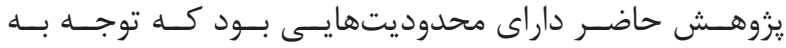

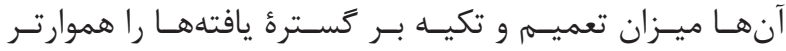

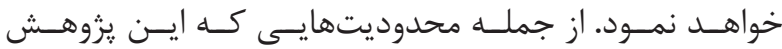

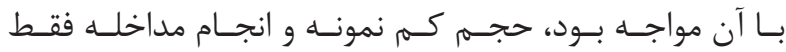

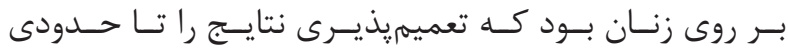

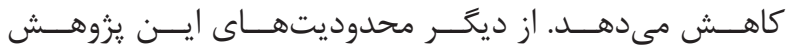

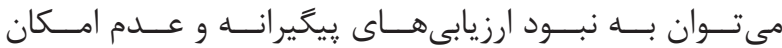

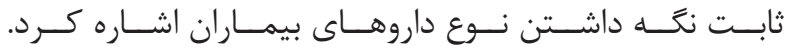

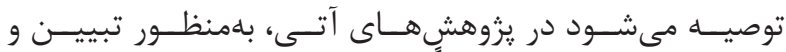

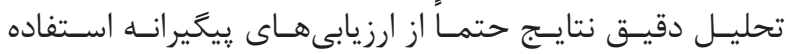

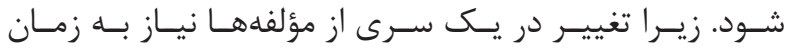

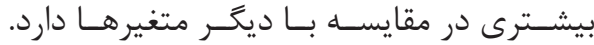

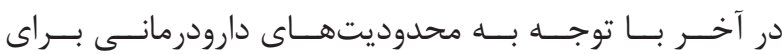

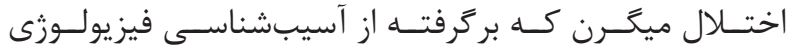

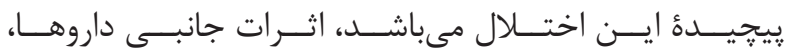

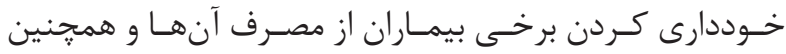

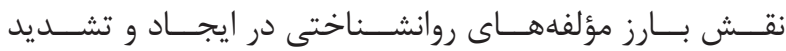

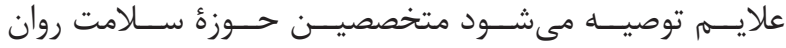

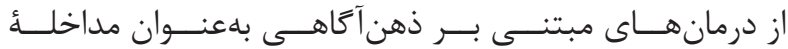

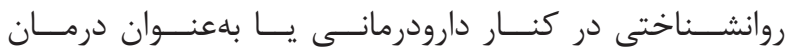

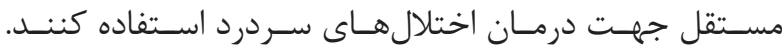

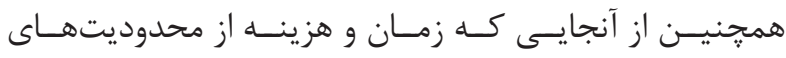

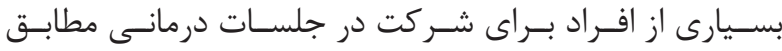

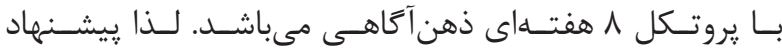

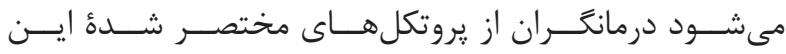

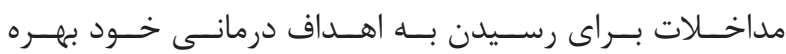

جوينــد.

تشكر و قدردانى

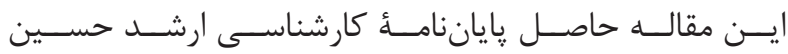

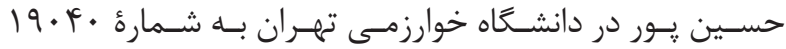

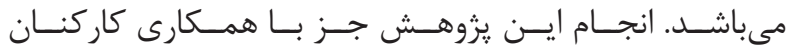

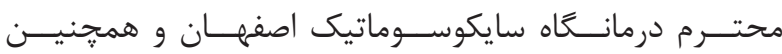

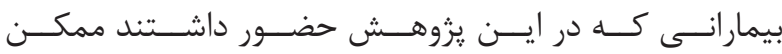

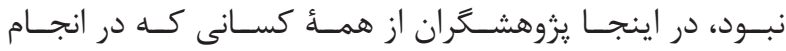

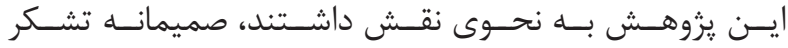

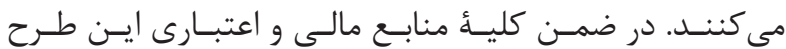

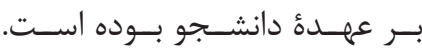

\section{${ }^{29}$ Carmody}

${ }^{30}$ Baer

${ }^{31}$ Ong

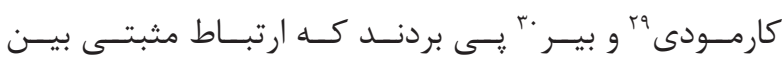

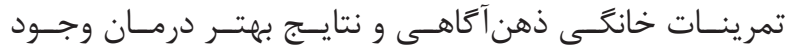

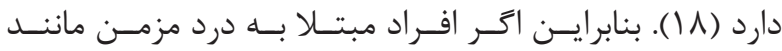

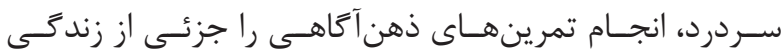

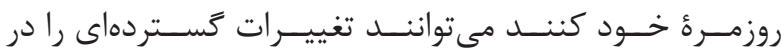

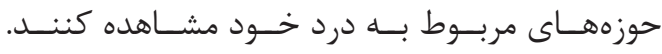

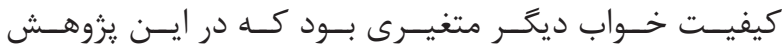

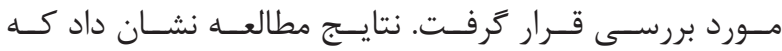

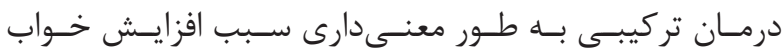

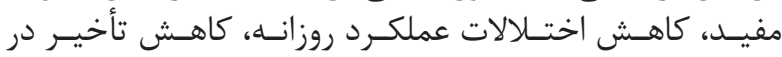

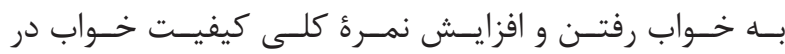

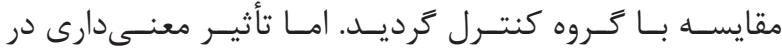

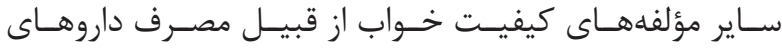

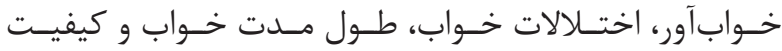

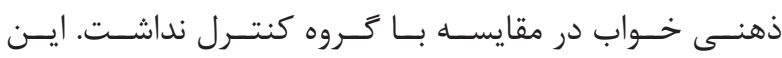

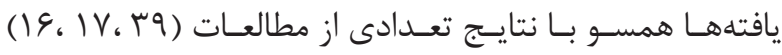

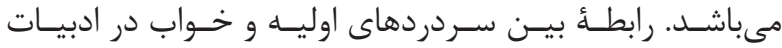

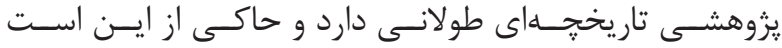

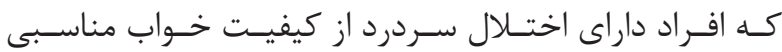

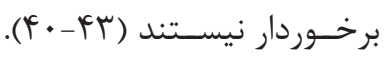

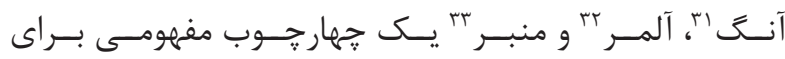

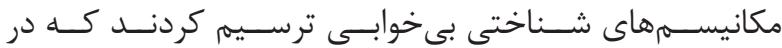

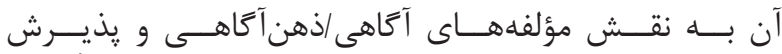

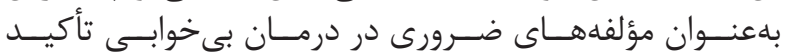

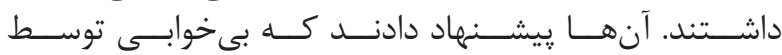

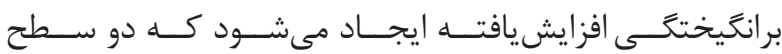

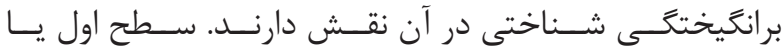

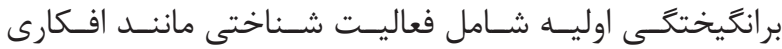

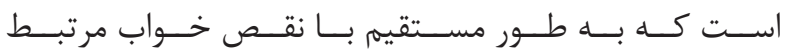

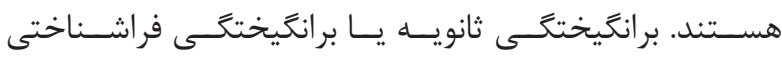

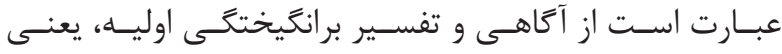

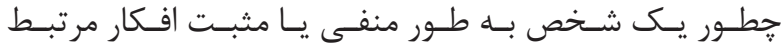

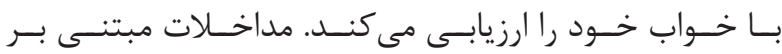

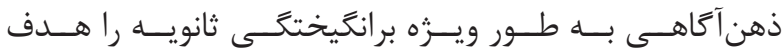

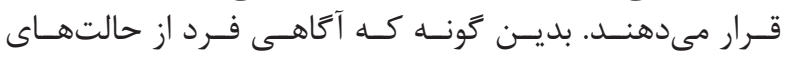

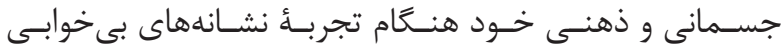

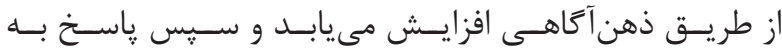

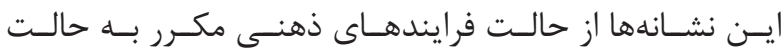

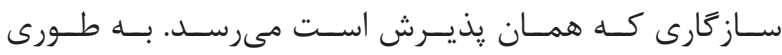

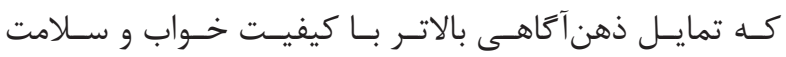

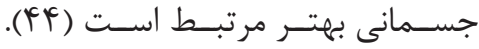

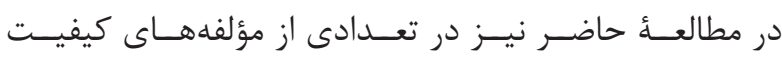

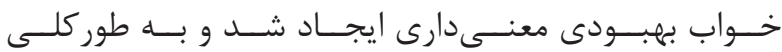

${ }^{32}$ Ulmer

${ }^{33}$ Manber 
1. Radtke A, Neuhauser H. Prevalence and burden of headache and migraine in Germany. Headache. 2009; 49(1): 79-89.

2. Pietrobon D, Moskowitz M. Pathophysiology of migraine. Annu Rev Physiol. 2013; 75(1): 365-91.

3. Alberti A. Headache and sleep. Sleep Med Rev. 2006; 10(6): 431-7.

4. Kelman L, Pains J. Headache and sleep: examination of sleep patterns and complaints in a large clinical sample of migraineurs. Headache. 2005; 45(7): 904-10.

5. Ziegler D, Murrow R. Headache. 2th ed. Philadelphia: JB Lippincott. 1988; p. 1-35.

6. Shamliyan TA, Choi JY, Ramakrishnan R, Miller JB, Wang SY, Taylor, FR, et al. Preventive pharmacologic treatments for episodic migraine in adults. J Gen Intern Med. 2013; 28(9): 1225-37.

7. Bigal M, Lipton R. The differential diagnosis of chronic daily headaches: An algorithm-based approach. J Headache Pain. 2007; 8(5): 263-72.

8. Gauthier J, Ivers H, Carrier S. Nonpharmacological approaches in the management of recurrent headache disorders and their comparison and combination with pharmacotherapy. Clin Psychol Rev. 1996; 16(6): 54371.

9. Wells R, Burch R, Paulsen R, Wayne P, Houle T, Loder E. Meditation for migraines: a pilot randomized controlled trial. Headache. 2014; 54(9): 1484-95.

10. Bishop S, Lau M, Shapiro S, Carlson L, Anderson N, Carmody J, et al. Mindfulness: A proposed operational definition. Clin Psychol. 2004; 11(3): 230-41.

11. Baer R, Smith G, Hopkins J, Krietemeyer J, Toney L. Using self-report assessment methods to explore facets of mindfulness. Assessment. 2006; 13(1): 27-45.

12. Cardaciotto L, Herbert J, Forman E, Moitra E, Farrow V. The assessment of present-moment awareness and acceptance: The philadelphia mindfulness scale. Assessment. 2008; 15(2): 204-23.

13. Rosenzweig S, Greeson J, Reibel D, Green J, Jasser S, Beasley D. Mindfulness-based stress reduction for chronic pain conditions: variation in treatment outcomes and role of home meditation practice. J Psychosom Res. 2010; 68(1): 29-36.

14. Morone N, Greco C, Weiner D. Mindfulness meditation for the treatment of chronic low back pain in older adults: A randomized controlled pilot study. Pain. 2008; 134(3): 310-9.

15. Kabat-Zinn J, Lipworth L, Burney R, Sellers W. Four-year follow-up of a meditation-based program for the self-regulation of chronic pain: treatment outcomes and compliance. Clin J Pain. 1986; 3(1): 159-73.

16. Bassaknezhad S, Aghajani Afjadi A, Zargar Y. The effectiveness of cognitive group therapy based on mindfulness on sleep quality and life quality in female university students. J Psychol Achv. 2016; 18(2): 181-9.

17. Black DS, O’Reilly GA, Olmstead R, Breen EC, Irwin MR. Mindfulness meditation and improvement in sleep quality and daytime impairment among older adults with sleep disturbances: A randomized clinical trial. JAMA Intern Med. 2015; 175(4): 494-501.

18. Carmody J, Baer R. How long does a mindfulnessbased stress reduction program need to be? A review of class contact hours and effect sizes for psychological distress. J Psychol. 2009; 65(6): 627-38.

19. Zeidan F, Gordon N, Merchant J, Goolkasian P. The effects of brief mindfulness meditation training on experimentally induced pain. J Pain. 2010; 11(3): 199209.

20. Kingston J, Chadwick P, Meron D, Skinner T. A pilot randomized control trial investigating the effect of mindfulness practice on pain tolerance, psychological well-being, and physiological activity. J Psychosom Res. 2007; 62(3): 297-300.

21. Bizarro L, Menezes C. Effects of a brief meditation training on negative affect, trait anxiety and concentrated attention. Paidéia. 2015; 25(62): 393-401.

22. Tang Y, Lu Q, Feng H, Tang R, Posner M. Short-term meditation increases blood flow in anterior cingulate cortex and insula. Front Psychol. 2015; 6: 212.

23. Tang YY, Ma Y, Wang J, Fan Y, Feng S, Lu Q, et al. Short-term meditation training improves attention and self-regulation. Proc Natl Acad Sci U S A. 2007; 104(43): 17152-6.

24. Cathcart S, Galatis N, Immink M, Proeve M, Petkov J. Brief mindfulness-based therapy for chronic tensiontype headache: A randomized controlled pilot study. Behav Cogn Psychother. 2014; 42(1): 1-15.

25. First MB, Spitzer RL, Gibbon M, Williams, JB. Structural clinical interview for DSM-IV-TR axis 


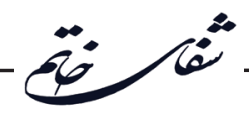

I disorders (SCID-I). Washington, DC: American Psychiatric Press. 1996.

26. Tran GQ, Smith GP. Behavioral assessment in the measurement of treatment outcome. Haynes SN, Heiby EM. Comprehensive handbook of psychological assessment. New York, NY: Wiley. 2004; p. 269-290.

27. Sharifi V, Asadi S, Mohammadi MR, Amin H, Kaviani H, Semnani Y, et al. Reliability and feasibility of the persian version of the structured diagnostic interview for DSM-IV (SCID). Adv Cogn Sci. 2004; 6(1-2): 10-22.

28. Stewart W, Lipton R, Dowson A, Sawyer J. Development and testing of the Migraine Disability Assessment (MIDAS) Questionnaire to assess headacherelated disability. Neurology. 2001; 56(6): 20-8.

29. Mo'tamedi H, Rezaiemaram P, Tavallaei R. The effectiveness of a group-based acceptance and commitment additive therapy on rehabilitation of female outpatients with chronic headache: Preliminary findings reducing 3 dimensions of headache impact. Headache. 2002; 52(7): 1106-19.

30. Aloba O, Adewuya A, Ola B, BMM. Validity of the Pittsburgh sleep quality index (PSQI) among nigerian university students. Sleep Med. 2007; 8(3): 266-70.

31. Burkhulter H, Sereika M, Engberig S, Justrice A, Steriger J, Geest S. Structure validity of the Pittsburgh Sleep Quality Index in renal transplant recipients: A confirmatory factor analysis. Sleep Biol Rhythms. 2010; 8: $274-81$.

32. Asadnia S, Sepehrian Azar F, Saadatmand S, Mosarrezaii Aghdam A. The relationship between sleep qualities with migraine headaches among Urmia university students. Urmia University Medical Sciences. 2013; 24(4): 286-94.

33. Rouleau C, Garland SN, Carlson LE. The impact of mindfulness-based interventions on symptom burden, positive psychological outcomes, and biomarkers in cancer patients. Cancer Manag Res. 2015; 7: 121-31.

34. Schmidt S, Simshäuser K, Aickin M, Lüking M, Schultz C, Kaube H. Mindfulness-based stress reduction is an effective intervention for patients suffering from
migraine-Results from a controlled trial. Eur J Integr Med. 2010; 2(4): 196.

35. Rosdahl DR. The effect of mindfulness meditation on tension headaches and secretory. [doctoral dissertation]. arizona, USA: University of Arizona. 2003. http:// arizona.openrepository.com.

36. Day M, Thorn B, Ward L, Rubin N, Hickman S, Scogin F, et al. Mindfulness-based cognitive therapy for the treatment of headache pain: a pilot study. Clin J Pain. 2014; 30(2): 152-61.

37. La Cour P, Petersen M. Effects of mindfulness meditation on chronic pain: a randomized controlled tria. Pain Med. 2015; 16(4): 641-52.

38. Pradhan E, Baumgarten M, Langenberg P, Handwerger B, Gilpin A, Berman B, et al. Effect of mindfulness based stress reduction in rheumatoid arthritis patients. Arthritis Care Res. 2007; 57(7): 1134-42.

39. Gong H, Ni C, Liu Y, Zhang Y, Su W, Lian Y, et al. Mindfulness meditation for insomnia: A meta-analysis of randomized controlled trials. J Psychosom Res. 2016; 89: 1-6.

40. Miller VA, Palermo TM, Powers SW, Scher MS, Hershey AD. Migraine headaches and sleep disturbances in children. Headache. 2003; 43(4): 362-8.

41. Delgado E, Schmidt J, Carlson C, Deleeuw R, Okeson J. Psychological and sleep quality differences between chronice daily headache and temporomandibular disorders patients. Cephalagia. 2004; 24(6): 446-54.

42. Seidel S, Hartl T, Weber M, Matterey S, Paul A, Riederer F, et al. Quality of sleep, fatigue and daytime sleepiness in migraine - a controlled study. Cephalalgia. 2009; 29(6): 662-9.

43. Safarinia M, Shahandeh M, AliAkbari-Dehkordi M, Bagherian-Sararoudi R. The effect of psychosocial group-therapy on improvement of migraine attacks and quality of life of patients with migraine. Journal of Isfahan Medical Sciences. 2015; 32(319): 2401-10.

44. Ong J, Ulmer C, Manber R. Improving sleep with mindfulness and acceptance: A metacognitive model of insomnia. Behav Res Ther. 2012; 50(11): 651-60. 